\title{
Analysis of Association of Occupational Physical Activity, Leisure-Time Physical Activity, and Sedentary Lifestyle with Hypertension according to the Adherence with Aerobic Activity in Women Using Korea National Health and Nutrition Examination Survey 2016-2017 Data
}

\author{
Mikyung Ryu, ${ }^{1,2}$ Sol Lee, ${ }^{3,4}$ Ho Gym, ${ }^{3,5}$ Weon-Chil Baek, ${ }^{1}$ and Heejin Kimm ${ }^{6}{ }^{6}$ \\ ${ }^{1}$ Department of Sports and Health Science, College of Human-Centered Convergence, Kyonggi University, Suwon, \\ Republic of Korea \\ ${ }^{2}$ Institute on Aging, Ajou University Medical Center, Suwon, Republic of Korea \\ ${ }^{3}$ Department of bbko Research Center, bbko Big-Data R\&D, Seoul, Republic of Korea \\ ${ }^{4}$ Department of Health Policy and Management, Korea University, Seoul, Republic of Korea \\ ${ }^{5}$ Department of Epidemiology and Health Promotion, Graduate School of Public Health, Yonsei University, Seoul, \\ Republic of Korea \\ ${ }^{6}$ Department of Epidemiology and Health Promotion, Institute for Health Promotion, Graduate School of Public Health, \\ Yonsei University, Seoul, Republic of Korea
}

Correspondence should be addressed to Heejin Kimm; heejink@yuhs.ac

Received 13 August 2019; Revised 7 November 2019; Accepted 10 January 2020; Published 14 February 2020

Academic Editor: Franco Veglio

Copyright (C) 2020 Mikyung Ryu et al. This is an open access article distributed under the Creative Commons Attribution License, which permits unrestricted use, distribution, and reproduction in any medium, provided the original work is properly cited.

Purpose. We investigated the association between occupational physical activity, leisure-time physical activity, and sedentary lifestyle with hypertension by adherence with aerobic exercise in middle-aged and elderly women. Methods. A cross-sectional analysis was performed using Korea National Health and Nutrition Examination Survey (KNHANES), a nationally representative data between 2016 and 2017. A total of 4,241 women aged 40 years or older were included. Hypertension diagnosed by physician and exercise status was asked by questionnaires. Results. Mean age of the participants was 58.4 ( \pm 11.4 , range: $40 \sim 80$ years). There were $1,681(39.6 \%)$ women in the aerobic activity adherence group. In the logistic regression analysis with adjustment for confounding factors, frequency of occupational physical activity (OPA) level (OR 1.931; $p=0.048$, in $\leq 4$ per week group), walking frequency (OR 0.436; $p=0.001$, in 5 7 days per week walking group compared with never walking group) in the aerobic activity adherence group, sitting hours (OR 1.849; 95\% CI: 1.279-2.673, $p=0.001$, in 13 hours or longer group compared with 6 hours or less sitting hours group), and muscle strengthening exercises (OR 0.554; 95\% CI: 0.353-0.870, $p=0.010$, $1 \sim 4$ days per week compared with never) in the nonadherence group were significantly associated with hypertension. Conclusions. In the aerobic activity adherence group, further research is needed to identify the influence of occupational physical activity. In the aerobic activity nonadherence group, decreasing sitting hours and increasing endurance exercise may be helpful.

\section{Introduction}

Hypertension has been a well-known condition causing cardiovascular disease, stroke, and chronic kidney disease [1-3], leading to premature mortality and finally death if not detected and treated within the appropriate period [4]. Hypertension is also a worldwide important public health issue and epidemiological matter [5]. Moreover, it has been reported that health-related quality of life is lower in people with diagnosed hypertension than those without diagnosed 
hypertension [6]. Moreover, the estimated number of adults with hypertension has increased over the years from 594 million in 1975 to 1.13 billion in 2015 [7]. Physical activity is known to be associated with decreased incidence of hypertension. It has showed benefits on blood pressure not only in people with normal blood pressure but also in patients with prehypertension or hypertension [8].

Physical training, particularly aerobic exercises, is recommended as an antihypertensive solution leading to significant blood pressure, body weight, body fat, and waist circumference reduction, along with insulin sensitivity and HDL cholesterol level improvement [9, 10]. Also, regular aerobic training has been reported to reduce blood pressure in both younger and older people with hypertension by decreasing aortic stiffness and enhanced flow-mediated arterial dilation mediated by increased nitric oxide released from endothelial cells lining these blood vessels, similar to blood pressure reductions by a single antihypertensive drug [11]. In previous studies, regular physical training such as walking, jogging, or swimming for 30 to 45 minutes can reduce blood pressure and is recommended by current American and European hypertension guidelines [12, 13]. These were leisure-time physical activity, which consistently showed a positive effect on cardiovascular outcomes, including hypertension $[14,15]$. The negative consequences of sedentary lifestyles are also well established [16].

However, despite the obvious reduction of cardiovascular mortality in other reports [15], there has been controversy among studies on the effects of occupational physical activity due to the risk of anaerobic components or injuries, depending on the nature of the work $[14,17]$. Few studies explored the association of occupational, leisure-time, and sedentary activities with hypertension diagnosed by physician in middleaged and elderly women. It is unclear whether this association depends on the adherence with aerobic activity.

The aim of this study is to examine the association of occupational physical activity, leisure-time physical activity, and sedentary lifestyle with hypertension according to the adherence with aerobic exercise in a nationally representative sample of women over 40 years of age, using recent Korea National Health and Nutrition Examination Survey (KNHANES) Data.

\section{Methods}

2.1. Participants. In this study, a cross-sectional analysis was performed using the Korea National Health and Nutrition Examination Survey (KNHANES) data collected by the Korea Centers for Disease Control and Prevention (KCDC) from 2016 to 2017. KNHANES is a surveillance system to assess the health and nutritional status of population in the Republic of Korea.

KNHANES includes a health interview, health examination, and nutrition survey. The interview and examination are performed by trained medical staff and interviewers. A multistage stratified cluster sampling was done for the household unit selection. The Institutional Review Board of the KCDC approved the data collection with written informed consent forms from all participants. The details of the survey design and data resource were described on a profiles paper in 2014 [18]. In this study, women participants older than 40 years of age who completed both answers on hypertension diagnosis (yes or no) and aerobic activities (yes or no) were included $(n=4,241)$.

2.2. Variables. Hypertension was defined as "participants' answer on hypertension diagnosed by medical doctor" from health survey questionnaires. Participants with no response were excluded from the analysis. If the participants answered "yes" to the questions for moderate intensity physical activity (at least 2.5 hours per week) or "yes" for vigorous intensity physical activity (at least 1.5 hours per week), they were classified as "aerobic activity adherence group." Those who failed to be classified as the adherence group were categorized as "nonadherence group." If the participants answered "yes" to the questions for muscle strengthening exercises (MSE) such as push-up, sit-up, dumbbell, or other types of muscle exercises more than 2 days for the past week, they were classified as "adherence group."

Sociodemographic characteristics including age, quartiles of equivalent income, and education (less than elementary school, less than middle school, less than high school, and more than university graduation) were included in the questionnaires. Smoking status was classified as never smoker, former smoker, or current smoker, and alcohol consumption was divided into 4 categories (never, no drink within recent 1 year, less than 4 times drink per a month, or more than 5 times per month). Sleeping hours were classified as 7 hours of sleep per day in weekdays as well as in weekends, respectively. For physical activities, each of vigorous or moderate intensity physical activity group was divided into two aspects, work (occupational physical activity, OPA) and leisure (leisure-time physical activity, LPA), with 3 subcategories (never, $\leq 4$ days per week, or $5 \sim 7$ days per week), respectively. Sitting hours were classified as $\leq 6$, $7 \sim 12$, or $\geq 13$ hours per a day. Frequency of walking per week was divided into 3 categories (never, $\leq 4$ days per week, or 5 7 days per week).

2.3. Statistical Analysis. The data were expressed by numbers and percentages for describing the general characteristics of the study participants. The frequency in the adherence group and the nonadherence group was compared by the chisquare test. To determine the difference for clinical variables in each group, independent two-sample $t$-test was done. Odds ratios (ORs) and 95\% confidence intervals (CIs) using binomial logistic regression were obtained to analyze an association between physical activity and hypertension in women with adjustment for other covariates. A $p$ value less than 0.05 was used for statistical significance. IBM SPSS statistics 22.0 software (IBM Corp, Armonk, NY) was used.

\section{Results}

3.1. General Characteristics of Study Population. The mean age of the 4,241 women participants from KNHANES 20162017 data was 58.4 ( \pm 11.4 , range: $40-80$ years old). Among 
them, $29.1 \%(1,234)$ of participants had hypertension and $92.7 \%$ were never smokers. There were 1,681 (39.6\%) women in the aerobic activity adherence group and 2,560 (60.4\%) participants were in the nonadherence group. For muscle strengthening exercises adherence, 3,560 (83.9\%) participants never did the exercise, 465 (11.0\%) did 1-4 day per week, and 215 (5.1\%) did more than 5 days per week. Most participants reported no vigorous intensity physical activity for both occupation (99.5\%) and leisure time (94.6\%). There was a similar trend for moderate intensity physical activity (Table 1).

\subsection{General Characteristics and Physical Activity Status due to} Aerobic Activity Adherence. Aerobic activity adherence showed a significant association with age groups (40-49, 50-59, 60-69, and more than 70 years) (Table 2). Quartiles of equivalent income and education were associated with aerobic activity adherence $(p<0.05)$, and hypertension also showed a significant association with aerobic activity adherence. OPA and LPA days per week were significantly associated with aerobic activity adherence $(p<0.05)$. There was a significant association between aerobic activity adherence and physical activity days with moving location. Sitting hours were significantly associated with aerobic activity adherence $(p<0.05)$. Sleeping hours per week were associated with aerobic activity adherence $(p<0.05)$. However, no association was found between sleeping hours during weekend and aerobic activity adherence. Alcohol consumption showed significant association aerobic activity adherence $(p<0.05)$, but smoking status showed no association.

3.3. Comparison of Clinical Characteristics according to the Aerobic Activity Adherence. Systolic blood pressure (SBP) and diastolic blood pressure (DBP) showed significant differences between the aerobic activity nonadherence group and aerobic activity adherence group $(p<0.05)$. Body mass index (BMI) and HDL cholesterol showed significant differences between the two groups $(p<0.05)$. In triglyceride, glutamate oxaloacetate transaminase (GOT), and glutamatepyruvate transaminase (GPT) levels, there were significant differences between the two groups $(p<0.05)$. However, total cholesterol showed no difference (Table 3 ).

\subsection{Association between Physical Activity and Hypertension in} the Aerobic Activity Nonadherence Group. In the aerobic activity nonadherence group, the odds ratio (OR) for hypertension in muscle strengthening exercises (MSE) adherence group (1 4 days per week) was 0.468 (95\% CI: $0.322-0.681, p<0.001)$ in the unadjusted model and 0.554 (95\% CI: $0.353-0.870)$ in the adjusted model with adjustment for age, quartiles of equivalent income, education levels, smoking status, and alcohol consumption, compared with the "never" group.

For sitting hours, ORs for hypertension were 1.211 (95\% CI: $1.008-1.455)$ in 7 12 hours group and 2.275 (95\% CI: 1.704-3.036) in 13 hours or longer group, compared with 6 hours or less sitting hours group in unadjusted model, respectively. They were 1.161 (95\% CI: 0.929-1.451) and 1.849
(95\% CI: 1.279-2.673) compared with 6 hours or less sitting hours group in the adjusted model with adjustment of age, quartiles of equivalent income, education levels, smoking status, and alcohol consumption, respectively.

A significant association was found between hypertension and days of walking per week. The OR for hypertension was 0.802 (95\% CI: 0.644-0.998) in $\leq 4$ days per week group, compared with the never walking group in the unadjusted model. However, no significant association was found with hypertension after adjustment.

Sleeping hours during week or weekend showed significant association with hypertension in the unadjusted model. However, the association disappeared in results from the adjusted model (Table 4 ).

\subsection{Association between Physical Activity and Hypertension in} the Aerobic Activity Adherence Group. In the aerobic activity adherence group who answered "yes" to the question about aerobic activity adherence, there was no significant association between MSE adherence and hypertension in both unadjusted model and adjusted model with adjustment forage, quartiles of equivalent income, education levels, smoking status, and alcohol consumption.

Frequency of leisure-time physical activity (LPA) level of vigorous and moderate intensity was associated with hypertension in the unadjusted model. The OR for hypertension was $0.537(p=0.030)$ in $\leq 4$ per week group and $0.314(p=0.017)$ in $5 \sim 7$ per week group of vigorous intensity, respectively. They were $0.701(p=0.032)$ in $\leq 4$ per week group of moderate intensity, but it was not significant in 5 7 per week group. The association was not significant in adjusted models.

Frequency of occupational physical activity (OPA) level of moderate intensity showed increased association with hypertension in the adjusted model (in $\leq 4$ per week group, $\mathrm{OR}=1.931 ; p=0.048)$.

Days of walking per week were significantly associated with hypertension. The OR for hypertension was 0.410 (95\% CI: $0.243-0.692, p=0.001)$ in $\leq 4$ per week group and 0.436 (95\% CI: 0.265-0.718) in 5 7 days per week group compared with the never walking group in the unadjusted model, respectively.

They were 0.532 (95\% CI: $0.284-0.997)$ and 0.530 (95\% CI: 0.292-0.963) compared with the never walking group in the adjusted model with adjustment of age, quartiles of equivalent income, education levels, smoking status, and alcohol consumption, respectively.

However, sitting hours per day and sleeping hours during week showed no significant association with hypertension in each model (Table 5).

\section{Discussion}

In the aerobic activity nonadherence group, women who had sedentary lifestyle such as long sitting hours showed increased association with hypertension and women who showed endurance and muscle strengthening exercise adherence were associated with decreased odds ratios of 
TABLE 1: Characteristics of the women participated in this study.

\begin{tabular}{|c|c|c|c|}
\hline Items & & $n$ & $\%$ \\
\hline \multirow{4}{*}{ Age (years) } & $40-49 y$ & 1148 & 27.1 \\
\hline & $50-59 y$ & 1210 & 28.5 \\
\hline & $60-69 y$ & 1042 & 24.6 \\
\hline & $\geq 70 y$ & 841 & 19.8 \\
\hline \multirow{4}{*}{ Income level } & Level 1 & 940 & 22.2 \\
\hline & Level 2 & 1061 & 25.0 \\
\hline & Level 3 & 1091 & 25.7 \\
\hline & Level 4 & 1142 & 26.9 \\
\hline \multirow{4}{*}{ Education level } & Elementary & 1355 & 32.0 \\
\hline & Middle & 568 & 13.4 \\
\hline & High & 1276 & 30.1 \\
\hline & University & 1036 & 24.4 \\
\hline \multirow{2}{*}{ Hypertension } & No & 3007 & 70.9 \\
\hline & Yes & 1234 & 29.1 \\
\hline \multirow{2}{*}{ Aerobic activity adherence ${ }^{a}$ (per week) } & No & 2560 & 60.4 \\
\hline & Yes & 1681 & 39.6 \\
\hline \multirow{3}{*}{ Muscle strengthening exercises adherence ${ }^{\mathrm{b}}$ (per week) } & Never & 3560 & 83.9 \\
\hline & $\leq 4$ & 465 & 11.0 \\
\hline & 5 7 days & 215 & 5.1 \\
\hline \multirow{3}{*}{ OPA vigorous intensity (per week) } & Never & 4219 & 99.5 \\
\hline & $\leq 4$ & 12 & 0.3 \\
\hline & 5 7 days & 10 & 0.2 \\
\hline \multirow{3}{*}{ OPA moderate intensity (per week) } & Never & 4061 & 95.8 \\
\hline & $\leq 4$ & 108 & 2.5 \\
\hline & $5 \sim 7$ days & 72 & 1.7 \\
\hline \multirow{3}{*}{ Physical activity days with moving location (per week) } & Never & 1801 & 42.5 \\
\hline & $\leq 4$ & 1101 & 26.0 \\
\hline & $5 \sim 7$ days & 1339 & 31.6 \\
\hline \multirow{3}{*}{ LPA vigorous intensity (per week) } & Never & 4011 & 94.6 \\
\hline & $\leq 4$ & 162 & 3.8 \\
\hline & $5 \sim 7$ days & 68 & 1.6 \\
\hline \multirow{3}{*}{ LPA moderate intensity (per week) } & Never & 3436 & 81.0 \\
\hline & $\leq 4$ & 577 & 13.6 \\
\hline & 5 7 days & 228 & 5.4 \\
\hline \multirow{3}{*}{ Sitting hours (per day) } & $\leq 6$ hours & 1831 & 43.2 \\
\hline & $\leq 12$ hours & 2028 & 47.8 \\
\hline & $\geq 13$ hours & 382 & 9.0 \\
\hline \multirow{3}{*}{ Walking (days per week) } & Never & 823 & 19.4 \\
\hline & $\leq 4$ days & 1578 & 37.2 \\
\hline & $\geq 5$ days & 1838 & 43.3 \\
\hline \multirow{2}{*}{ Sleeping hours in weekdays (per week) } & $<7$ hours & 2594 & 61.2 \\
\hline & $\geq 7$ hours & 1647 & 38.8 \\
\hline \multirow{2}{*}{ Sleeping hours in weekends (per week) } & $<7$ hours & 1989 & 46.9 \\
\hline & $\geq 7$ hours & 2252 & 53.1 \\
\hline \multirow{4}{*}{ Alcohol consumption (within 1 year) } & No experience & 884 & 20.8 \\
\hline & No drink within recent 1 year & 834 & 19.7 \\
\hline & $\leq 4$ times (months) & 2147 & 50.6 \\
\hline & $\geq 5$ times (months) & 376 & 8.9 \\
\hline \multirow{3}{*}{ Smoking status } & Never & 3933 & 92.7 \\
\hline & Former smoker & 162 & 3.8 \\
\hline & Current smoker & 145 & 3.4 \\
\hline
\end{tabular}

${ }^{a}$ Aerobic activity adherence: 2.5 -hour moderate intensity physical activity per week or 1.25 -hour vigorous intensity physical activity per week. ${ }^{\mathrm{b}}$ Muscle strengthening exercises adherence: push-up, sit-up, dumbbell, or other types of muscle exercises more than 2 days during 1 week recently. OPA: occupational physical activity; LPA: leisure-time physical activity. 
TABLE 2: General characteristics and physical activity status due to aerobic activity adherence.

\begin{tabular}{|c|c|c|c|c|c|c|c|}
\hline & \multirow[t]{2}{*}{ Items } & \multirow{2}{*}{$\begin{array}{c}\text { Total } \\
n\end{array}$} & \multicolumn{2}{|c|}{$\begin{array}{l}\text { Nonaerobic } \\
(n=2,560)\end{array}$} & \multicolumn{2}{|c|}{$\begin{array}{c}\text { Aerobic } \\
(n=1,681)\end{array}$} & \multirow[t]{2}{*}{$p$ value } \\
\hline & & & $n$ & $\%$ & $n$ & $\%$ & \\
\hline \multirow{4}{*}{ Age (years) } & $40-49 y$ & 1148 & 624 & 24.4 & 524 & 31.2 & \multirow{4}{*}{$<0.001^{* *}$} \\
\hline & $50-59 y$ & 1210 & 660 & 25.8 & 550 & 32.7 & \\
\hline & $60-69 y$ & 1042 & 640 & 25.0 & 402 & 23.9 & \\
\hline & $\geq 70 y$ & 841 & 636 & 24.8 & 205 & 12.2 & \\
\hline \multirow{4}{*}{ Income level } & Level 1 & 940 & 611 & 23.9 & 329 & 19.6 & \multirow{4}{*}{$<0.001^{* *}$} \\
\hline & Level 2 & 1061 & 660 & 25.8 & 401 & 23.9 & \\
\hline & Level 3 & 1091 & 640 & 25.1 & 451 & 26.8 & \\
\hline & Level 4 & 1142 & 643 & 25.2 & 499 & 29.7 & \\
\hline \multirow{4}{*}{ Education level } & Elementary & 1355 & 970 & 38.0 & 385 & 22.9 & \multirow{4}{*}{$<0.001^{* *}$} \\
\hline & Middle & 568 & 351 & 13.7 & 217 & 12.9 & \\
\hline & High & 1276 & 703 & 27.5 & 573 & 34.1 & \\
\hline & University & 1036 & 531 & 20.8 & 505 & 30.1 & \\
\hline \multirow{2}{*}{ Hypertension } & No & 3007 & 1689 & 66.0 & 1318 & 78.4 & \multirow{2}{*}{$<0.001^{* *}$} \\
\hline & Yes & 1234 & 871 & 34.0 & 363 & 21.6 & \\
\hline \multirow{3}{*}{$\begin{array}{l}\text { Aerobic activity } \\
\text { adherence }^{\text {a }} \text { (per week) }\end{array}$} & No & 3560 & 2265 & 88.5 & 1295 & 77.0 & \multirow{3}{*}{$<0.001^{* *}$} \\
\hline & Yes & 465 & 199 & 7.8 & 266 & 15.8 & \\
\hline & Never & 215 & 95 & 3.7 & 120 & 7.1 & \\
\hline \multirow{3}{*}{$\begin{array}{l}\text { OPA vigorous } \\
\text { intensity (per week) }\end{array}$} & $\leq 4$ days & 4219 & 2557 & 99.9 & 1662 & 98.9 & \multirow{3}{*}{$<0.001^{* *}$} \\
\hline & 5 7 days & 12 & 2 & 0.1 & 10 & 0.6 & \\
\hline & Never & 10 & 1 & 0.0 & 9 & 0.5 & \\
\hline \multirow{3}{*}{$\begin{array}{l}\text { OPA moderate } \\
\text { intensity (per week) }\end{array}$} & $\leq 4$ days & 4061 & 2533 & 98.9 & 1528 & 90.9 & \multirow{3}{*}{$<0.001^{* *}$} \\
\hline & 5 7 days & 108 & 25 & 1.0 & 83 & 4.9 & \\
\hline & Never & 72 & 2 & 0.1 & 70 & 4.2 & \\
\hline \multirow{3}{*}{$\begin{array}{l}\text { Physical activity days } \\
\text { with moving location } \\
\text { (per week) }\end{array}$} & $\leq 4$ days & 1801 & 1592 & 62.2 & 209 & 12.4 & \\
\hline & 5 7 days & 1101 & 710 & 27.7 & 391 & 23.3 & $<0.001^{* *}$ \\
\hline & Never & 1339 & 258 & 10.1 & 1081 & 64.3 & \\
\hline & $\leq 4$ days & 4011 & 2550 & 99.6 & 1461 & 86.9 & \\
\hline $\begin{array}{l}\text { LPA vigorous } \\
\text { intensity (per week) }\end{array}$ & $5 \sim 7$ days & 162 & 10 & 0.4 & 152 & 9.0 & $<0.001^{* *}$ \\
\hline & Never & 68 & 0 & 0.0 & 68 & 4.0 & \\
\hline & $\leq 4$ days & 3436 & 2412 & 94.2 & 1024 & 60.9 & \\
\hline $\begin{array}{l}\text { LPA moderate } \\
\text { intensity (ner week) }\end{array}$ & 5 7 days & 577 & 138 & 5.4 & 439 & 26.1 & $<0.001^{* *}$ \\
\hline intensity (per week) & Never & 228 & 10 & 0.4 & 218 & 13.0 & \\
\hline & $\leq 6$ hours & 1831 & 1003 & 39.2 & 828 & 49.3 & \\
\hline Sitting hours (per day) & $\leq 12$ hours & 2028 & 1290 & 50.4 & 738 & 43.9 & $<0.001^{* *}$ \\
\hline & $\geq 13$ hours & 382 & 267 & 10.4 & 115 & 6.8 & \\
\hline & Never & 823 & 731 & 28.6 & 92 & 5.5 & \\
\hline Walking (days per week) & $\leq 4$ days & 1578 & 1126 & 44.0 & 452 & 26.9 & $<0.001^{* *}$ \\
\hline & 5 7 days & 1838 & 702 & 27.4 & 1136 & 67.6 & \\
\hline Sleeping hours in & $<7$ hours & 2594 & 1505 & 58.8 & 1089 & 64.8 & $0.001^{* *}$ \\
\hline weekdays (per week) & $\geq 7$ hours & 1647 & 1055 & 41.2 & 592 & 35.2 & $<0.001$ \\
\hline Sleeping hours in & $<7$ hours & 1989 & 1189 & 46.4 & 800 & 47.6 & 0.465 \\
\hline weekends (per week) & $\geq 7$ hours & 2252 & 1371 & 53.6 & 881 & 52.4 & 0.465 \\
\hline & No experience & 884 & 584 & 22.8 & 300 & 17.8 & \\
\hline Alcohol consumption & No drink within recent 1 year & 834 & 535 & 20.9 & 299 & 17.8 & \\
\hline (within 1 year) & $\leq 4$ times (months) & 2147 & 1210 & 47.3 & 937 & 55.7 & $<0.001^{* *}$ \\
\hline & $\geq 5$ times (months) & 376 & 231 & 9.0 & 145 & 8.6 & \\
\hline & Never & 3933 & 2357 & 92.1 & 1576 & 93.8 & \\
\hline Smoking status & Former smoker & 162 & 103 & 4.0 & 59 & 3.5 & 0.091 \\
\hline & Current smoker & 145 & 99 & 3.9 & 46 & 2.7 & \\
\hline
\end{tabular}

${ }^{a}$ Aerobic activity adherence: 2.5 -hour moderate intensity physical activity per week or 1.25 -hour vigorous intensity physical activity per week. ${ }^{b}$ Muscle strengthening exercises adherence: push-up, sit-up, dumbbell, or other types of muscle exercises more than 2 days during 1 week recently. OPA: occupational physical activity; LPA: leisure-time physical activity. ${ }^{*} p<0.05 ;{ }^{* *} p<0.01$. 
TABLE 3: Comparison of clinical characteristics according to the aerobic activity adherence.

\begin{tabular}{|c|c|c|c|c|c|}
\hline & \multicolumn{2}{|c|}{$\begin{array}{c}\text { Aerobic activity } \\
\text { nonadherence group } \\
\quad(n=2,560)\end{array}$} & \multicolumn{2}{|c|}{$\begin{array}{l}\text { Aerobic activity adherence } \\
\quad \text { group }(n=1,681)\end{array}$} & \multirow[t]{2}{*}{$p$ value } \\
\hline & Mean & SD & Mean & SD & \\
\hline Mean SBP & 122.05 & 17.73 & 119.25 & 17.42 & $<0.001^{* *}$ \\
\hline Mean DBP & 74.58 & 9.51 & 75.17 & 9.32 & $0.048^{*}$ \\
\hline BMI & 24.12 & 3.57 & 23.79 & 3.34 & $0.002^{*}$ \\
\hline Total cholesterol & 198.51 & 39.27 & 199.82 & 38.15 & 0.294 \\
\hline HDL cholesterol & 52.48 & 12.53 & 54.61 & 12.66 & $<0.001^{* *}$ \\
\hline Triglyceride & 132.38 & 100.99 & 117.13 & 77.88 & $<0.001^{* *}$ \\
\hline AST (GOT) & 22.43 & 9.65 & 21.73 & 8.54 & $0.016^{*}$ \\
\hline ALT (GPT) & 19.85 & 12.98 & 18.84 & 12.08 & $0.011^{*}$ \\
\hline
\end{tabular}

SD: standard deviation; SBP: systolic blood pressure; DBP: diastolic blood pressure; BMI: body mass index; GOT: glutamate oxaloacetate transaminase; GPT: glutamate-pyruvate transaminase. ${ }^{*} p<0.05 ;{ }^{* *} p<0.01$.

TABLE 4: Association between physical activity and hypertension in the aerobic activity nonadherence group.

\begin{tabular}{|c|c|c|c|c|c|}
\hline Items & & Unadjusted OR & $p$ value & Adjusted $^{\mathrm{a}}$ OR & $p$ value \\
\hline $\begin{array}{l}\text { Muscle strengthening exercises adherence }{ }^{\mathrm{b}} \text { (per } \\
\text { week) }\end{array}$ & $\begin{array}{c}\text { Never } \\
\leq 4 \\
5 \sim 7 \text { days } \\
\end{array}$ & $\begin{array}{l}0.468 \\
0.830 \\
\end{array}$ & $\begin{array}{c}<0.001^{* *} \\
<0.001^{* *} \\
0.433\end{array}$ & $\begin{array}{l}0.554 \\
0.850 \\
\end{array}$ & $\begin{array}{c}0.034^{*} \\
0.010^{*} \\
0.570 \\
\end{array}$ \\
\hline OPA vigorous intensity (per week) & $\begin{array}{c}\text { Never } \\
\leq 4 \\
5 \sim 7 \text { days }\end{array}$ & 0.000 & $\begin{array}{l}1.000 \\
0.999 \\
1.000 \\
\end{array}$ & 0.000 & $\begin{array}{l}1.000 \\
0.999 \\
1.000 \\
\end{array}$ \\
\hline OPA moderate intensity (per week) & $\begin{array}{c}\text { Never } \\
\leq 4 \\
5 \sim 7 \text { days }\end{array}$ & $\begin{array}{l}0.446 \\
2.797\end{array}$ & $\begin{array}{l}0.247 \\
0.116 \\
0.570 \\
\end{array}$ & $\begin{array}{l}0.533 \\
1.577\end{array}$ & $\begin{array}{l}0.550 \\
0.282 \\
0.847\end{array}$ \\
\hline $\begin{array}{l}\text { Physical activity days with moving location (per } \\
\text { week) }\end{array}$ & $\begin{array}{c}\text { Never } \\
\leq 4 \\
5 \sim 7 \text { days }\end{array}$ & $\begin{array}{l}1.173 \\
1.301 \\
\end{array}$ & $\begin{array}{l}0.131 \\
0.134 \\
0.091\end{array}$ & $\begin{array}{l}1.000 \\
1.410\end{array}$ & $\begin{array}{l}0.173 \\
0.999 \\
0.069\end{array}$ \\
\hline LPA vigorous intensity (per week) & $\begin{array}{c}\text { Never } \\
\leq 4 \\
5 \sim 7 \text { days }\end{array}$ & 0.262 & 0.212 & 0.158 & 0.149 \\
\hline LPA moderate intensity (per week) & $\begin{array}{c}\text { Never } \\
\leq 4 \\
5 \sim 7 \text { days } \\
\end{array}$ & $\begin{array}{l}0.782 \\
1.247 \\
\end{array}$ & $\begin{array}{l}0.493 \\
0.254 \\
0.757 \\
\end{array}$ & $\begin{array}{l}1.318 \\
1.821\end{array}$ & $\begin{array}{l}0.441 \\
0.281 \\
0.477 \\
\end{array}$ \\
\hline Sitting hours (per day) & $\begin{array}{c}\leq 6 \text { hours } \\
7 \sim 12 \text { hours } \\
\geq 13 \text { hours }\end{array}$ & $\begin{array}{l}1.211 \\
2.275\end{array}$ & $\begin{array}{c}<0.001^{* *} \\
0.041^{*} \\
<0.001^{* *} \\
\end{array}$ & $\begin{array}{l}1.161 \\
1.849 \\
\end{array}$ & $\begin{array}{c}0.005^{*} \\
0.190 \\
0.001^{*} \\
\end{array}$ \\
\hline Walking (days per week) & $\begin{array}{c}\text { Never } \\
\leq 4 \\
5 \sim 7 \text { days } \\
\end{array}$ & $\begin{array}{l}0.802 \\
0.953\end{array}$ & $\begin{array}{c}0.096 \\
0.048^{*} \\
0.704 \\
\end{array}$ & $\begin{array}{l}1.187 \\
1.310 \\
\end{array}$ & $\begin{array}{l}0.203 \\
0.213 \\
0.078 \\
\end{array}$ \\
\hline Sleeping hours in weekdays (per week) & $\geq 7$ hours & 2.007 & $<0.001^{* *}$ & 1.329 & 0.065 \\
\hline Sleeping hours in weekends (per week) & $\geq 7$ hours & 0.591 & $<0.001^{* *}$ & 0.942 & 0.698 \\
\hline Alcohol consumption (within 1 year) & $\begin{array}{c}\text { No experience } \\
\text { No drink within recent } 1 \text { year } \\
\leq 4 \text { times (months) } \\
\geq 5 \text { times (months) }\end{array}$ & $\begin{array}{l}0.680 \\
0.491 \\
0.475 \\
\end{array}$ & $\begin{array}{c}<0.001^{* *} \\
0.002^{*} \\
<0.001^{* *} \\
<0.001^{* *}\end{array}$ & $\begin{array}{l}0.895 \\
0.912 \\
0.807\end{array}$ & $\begin{array}{l}0.776 \\
0.474 \\
0.497 \\
0.337\end{array}$ \\
\hline Smoking status & $\begin{array}{c}\text { Never } \\
\text { Former smoker } \\
\text { Current smoker }\end{array}$ & $\begin{array}{l}0.846 \\
0.690\end{array}$ & $\begin{array}{l}0.262 \\
0.465 \\
0.134 \\
\end{array}$ & $\begin{array}{l}0.776 \\
0.637\end{array}$ & $\begin{array}{l}0.246 \\
0.372 \\
0.144\end{array}$ \\
\hline
\end{tabular}

${ }^{a}$ Adjusted by covariates (controlling age, income level, education levels, smoking status, and alcohol consumption) in multiple logistic regression analysis. ${ }^{b}$ Muscle strengthening exercises adherence: push-up, sit-up, dumbbell, or other types of muscle exercises more than 2 days during 1 week recently. OR: odds ratio; OPA: occupational physical activity; LPA: leisure-time physical activity. ${ }^{*} p<0.05 ;{ }^{* *} p<0.01$. 
TABLE 5: Association between physical activity and hypertension in the aerobic activity adherence group.

\begin{tabular}{|c|c|c|c|c|c|}
\hline Items & & Unadjusted OR & $p$ value & Adjusted $^{\mathrm{a}} \mathrm{OR}$ & $p$ value \\
\hline \multirow{3}{*}{$\begin{array}{l}\text { Muscle strengthening exercises adherence }{ }^{b} \text { (per } \\
\text { week) }\end{array}$} & Never & & 0.879 & & 0.527 \\
\hline & $\leq 4$ & 0.928 & 0.693 & 1.292 & 0.259 \\
\hline & 5 7 days & 1.069 & 0.789 & 1.067 & 0.819 \\
\hline \multirow{3}{*}{ OPA vigorous intensity (per week) } & Never & & 0.541 & & 0.698 \\
\hline & $\leq 4$ & 0.890 & 0.891 & 0.558 & 0.572 \\
\hline & $5 \sim 7$ days & 0.300 & 0.270 & 0.422 & 0.516 \\
\hline \multirow{3}{*}{ OPA moderate intensity (per week) } & Never & & $0.050^{*}$ & & 0.123 \\
\hline & $\leq 4$ & 1.515 & 0.123 & 1.931 & $0.048^{*}$ \\
\hline & $5 \sim 7$ days & 0.509 & 0.074 & 0.859 & 0.725 \\
\hline \multirow{3}{*}{$\begin{array}{l}\text { Physical activity days with moving location (per } \\
\text { week) }\end{array}$} & Never & & 0.623 & & 0.861 \\
\hline & $\leq 4$ & 1.203 & 0.467 & 1.073 & 0.813 \\
\hline & 5 7 days & 1.269 & 0.331 & 1.153 & 0.619 \\
\hline \multirow{3}{*}{ LPA vigorous intensity (per week) } & Never & & $0.007^{*}$ & & 0.474 \\
\hline & $\leq 4$ & 0.537 & $0.030^{*}$ & 0.852 & 0.627 \\
\hline & $5 \sim 7$ days & 0.314 & $0.017^{*}$ & 0.538 & 0.251 \\
\hline \multirow{3}{*}{ LPA moderate intensity (per week) } & Never & & 0.101 & & 0.338 \\
\hline & $\leq 4$ & 0.701 & $0.032^{*}$ & 0.798 & 0.256 \\
\hline & $5 \sim 7$ days & 0.906 & 0.652 & 1.163 & 0.552 \\
\hline \multirow{3}{*}{ Sitting hours (per day) } & $\leq 6$ hours & & 0.323 & & 0.845 \\
\hline & 7 12 hours & 1.211 & 0.133 & 1.002 & 0.991 \\
\hline & $\geq 13$ hours & 1.102 & 0.692 & 0.841 & 0.576 \\
\hline \multirow{3}{*}{ Walking (days per week) } & Never & & $0.002^{*}$ & & 0.103 \\
\hline & $\leq 4$ & 0.410 & $0.001^{*}$ & 0.532 & $0.049^{*}$ \\
\hline & $5 \sim 7$ days & 0.436 & $0.001^{*}$ & 0.530 & $0.037^{*}$ \\
\hline Sleeping hours in weekdays (per week) & $\geq 7$ hours & 1.335 & 0.076 & 1.182 & 0.409 \\
\hline Sleeping hours in weekends (per week) & $\geq 7$ hours & 0.656 & $0.007^{*}$ & 0.844 & 0.380 \\
\hline \multirow{4}{*}{ Alcohol consumption (within 1 year) } & No experience & & $<0.001^{* *}$ & & 0.859 \\
\hline & No drink within recent 1 year & 0.756 & 0.135 & 1.134 & 0.580 \\
\hline & $\leq 4$ times (months) & 0.545 & $<0.001^{* *}$ & 1.049 & 0.806 \\
\hline & $\geq 5$ times (months) & 0.403 & $0.001^{*}$ & 0.863 & 0.662 \\
\hline \multirow{3}{*}{ Smoking status } & Never & & 0.394 & & 0.264 \\
\hline & Former smoker & 1.538 & 0.172 & 1.878 & 0.109 \\
\hline & Current smoker & 1.020 & 0.961 & 0.890 & 0.798 \\
\hline
\end{tabular}

${ }^{a}$ Adjusted by covariates (controlling age, income level, education levels, smoking status, and alcohol consumption) in multiple logistic regression analysis. ${ }^{\mathrm{b}}$ Muscle strengthening exercises adherence: push-up, sit-up, dumbbell, or other types of muscle exercises more than 2 days during 1 week recently. OR: odds ratio; OPA: occupational physical activity; LPA: leisure-time physical activity. ${ }^{*} p<0.05 ;{ }^{* *} p<0.01$.

hypertension. Particularly, they showed increased association with hypertension along with increment of sitting hours, which is closely related to the level of occupational physical activity.

Previous studies on the effects of occupational physical activity (OPA) on hypertension are inconsistent [19]. OPA has also been shown to lower the risk of hypertension [15]; on the contrary, in the report of increasing risk in black men, it is considered that research on various population groups is necessary in consideration of racial differences, which supports the necessity of this study [20]. In addition, allcause mortality has been reported as well as hypertension [21]. Potential hypothesis raised to date includes the risk of anaerobic components or injuries [14], adverse social determinants and demanding job characteristics [17], and motion characteristics of OPA which is not helpful for cardiopulmonary function [21].

On the other hand, the mechanism of cardiovascular diseases due to increased sitting hours due to occupation has been explained more clearly. Decreased local contraction of muscles interferes with lipoprotein lipase activity, leading to an increase in triglycerides and a decrease in high-density lipoprotein cholesterol [22]. It is not easy to measure heterogeneous types of OPAs and working environment for the mechanisms by which OPAs pose a risk of hypertension [17], but further research is needed for clarification.

In the aerobic activity adherence group, women doing occupational physical activity of moderate intensity showed increased association with hypertension. Frequency of walking, e.g., days of walking per week, was associated with decreased odds ratios of hypertension in this group.

The relationship between physical activity and hypertension has been reported many times. The previous studies also reported impacts on RCT interventions and risk of coronary heart disease. In the study of Twinamasiko, the mean systolic blood pressure was higher in the sedentary work style group [16]. In a recent meta-analysis of 26 RCTs on physical activities, it was reported that physical activities, 
even with higher intensity, were associated with reduction in blood pressure in the participants with hypertension [23-26]. Meeting the target of American Physical Activity Guideline resulted in a $14 \%$ reduced risk of coronary heart disease compared with inactive people in adults [8]. Also, there was a research identifying the fact that daily physical activity was associated with blood pressure particularly in women [27].

Several studies reported that the mechanisms leading to reducing blood pressure by physical activity were related to cardiac remodelling, endothelial function, decreased oxidative stress, and the inflammation syndrome [28-30]. The mechanism by which aerobic exercise training lowers blood pressure in hypertensive patients has been reported for some time, and the mechanism has been suggested to be related to the sympathetic nerve activity (SNA) [31]. However, evidence suggests that muscle SNA is associated with the central sympathetic nerve activity (SNA) [32]. After 4 months of moderate intensity aerobic exercise training in hypertensive patients with unsuccessful treatment, initial excessive central sympathetic activation decreased, accompanied by decreased muscle SNA and improved arterial baroreflex regulation of SNA [33]. Usual pharmacological approaches for essential hypertension have not focused on regulating central sympathetic outflow, and the emerging therapeutic strategies targeting neurogenic hypertension such as aerobic exercise training, weight loss, stress reduction, central sympatholytics such as moxonidine or rilmenidine, statins, and surgical interventions such as therapeutic renal sympathetic denervation have implications for clinical management of hypertension [32]. Walking was identified to be related to decreased risk of hypertension in participants who showed adherence to aerobic activity, while increased sitting hours could lead to increased risk of hypertension in the participants not adhered to the regular aerobic activity. In Hu's report (2007), hypertensive women had the benefit of reduced cardiovascular mortality from all the type of physical activities such as occupational, leisure-time and daily "walking or cycling to and from work." Because of these results, walking in women can be consistently recommended [15]. There have been a number of researches reporting that regular or chronic dynamic aerobic and resistance training would be effective on reductions in blood pressure of the patients with hypertension [34-36]. In addition to moderate-vigorous physical activity, the BP lowering effect of reducing sedentary hours through interrupting prolonged sitting was recently reported and "sit less" has been recommended [37]. When this "breaks in prolonged sitting" was performed with morning exercise, it was effective to control BP in obese older adults, particularly in women [38].

Also it was demonstrated that the largest isometric training resulted in reductions of blood pressure in hypertensive patients which was similar to aerobic exercise training $[34,39,40]$. Moreover, the combination of muscle exercises and aerobic activity could be effective in controlling blood pressure [41]. In this study, even the participants without aerobic activities nonadherence demonstrated that regular muscle exercise was associated with decreased risk of hypertension.

There are several limitations in interpreting the results of this study. First, it was not possible to demonstrate any causal relationship between physical activities and hypertension in women aged from 40 to 80 years because of the cross-sectional design of this study. Secondly, there was no significant association between leisure-time activities and hypertension in neither adherence nor nonadherence group. This may be due to the small number of active women in the nonadherence group. Finally, there was no consideration that it was surgical menopause or natural menopause. Information on the risky components in the work activities such as chemical, mechanical injury, or job stress was insufficient to analyze the association between occupational physical activity and hypertension. A more comprehensive study may be necessary to overcome those limitations.

\section{Conclusion}

In the aerobic activity adherence group, women doing occupational physical activity of moderate intensity showed increased association with hypertension. Further research that identifies the dangerous components in occupational physical activity is warranted. Walking seems to have benefits and be appropriate to be recommended. In the aerobic activity nonadherence group, women who had long sitting hours showed increased association with hypertension, and women who showed endurance exercise adherence were associated with decreased odds ratios of hypertension. Decreasing sitting hours and increasing endurance exercise may be helpful for this group.

\section{Data Availability}

The data used to support the findings of this study are available from the website of the Korea National Health and Nutrition Examination Survey (https://knhanes.cdc.go.kr/ knhanes/main.do).

\section{Disclosure}

The funding sources had no role in the design, implementation, analyses, interpretation of the data, or decision to submit results.

\section{Conflicts of Interest}

The authors declared no conflicts of interest.

\section{Authors' Contributions}

Mikyung Ryu and Ho Kim participated in the study concept, design, data analyses, interpretation of analyses, and writing of the manuscript. Heejin Kimm participated in the study concept design, data interpretation, and critical appraisal of the content of the paper. Sol Leeand Weon-Chil Baek participated in data analysis, the interpretation of analyses, and critical revision of the paper.

\section{Acknowledgments}

This research was supported by Basic Science Research Program through the National Research Foundation of Korea (NRF) funded by the Ministry of Education, Science and Technology (14245). 


\section{References}

[1] N. R. Poulter, D. Prabhakaran, and M. Caulfield, "Hypertension," The Lancet, vol. 386, no. 9995, pp. 801-812, 2015.

[2] M. H. Forouzanfar, A. Afshin, L. T. Alexander et al., "Global, regional, and national comparative risk assessment of 79 behavioural, environmental and occupational, and metabolic risks or clusters of risks, 1990-2015: a systematic analysis for the global burden of disease study 2015," The Lancet, vol. 388, no. 10053, pp. 1659-1724, 2016.

[3] D. T. Lackland and M. A. Weber, "Global burden of cardiovascular disease and stroke: hypertension at the core," Canadian Journal of Cardiology, vol. 31, no. 5, pp. 569-571, 2015.

[4] P. A. James, S. Oparil, B. L. Carter et al., "2014 evidence-based guideline for the management of high blood pressure in adults," JAMA, vol. 311, no. 5, pp. 507-520, 2014.

[5] K. Athanasakis, "The socioeconomic effects of uncontrolled hypertension," Current Vascular Pharmacology, vol. 16, no. 1, pp. 5-9, 2017.

[6] Q. Yao, C. Liu, Y. Zhang, and L. Xu, "Health-related quality of life of people with self-reported hypertension: a national cross-sectional survey in China," International Journal of Environmental Research and Public Health, vol. 16, no. 10, p. 1721, 2019.

[7] B. Zhou, J. Bentham, M. Di Cesare et al., "Worldwide trends in blood pressure from 1975 to 2015: a pooled analysis of 1479 population-based measurement studies with 19.1 million participants," The Lancet, vol. 389, no. 10064, pp. 37-55, 2017.

[8] K. L. Piercy and R. P. Troiano, "Physical activity guidelines for Americans from the US department of health and human services," Circulation: Cardiovascular Quality and Outcomes, vol. 11, no. 11, Article ID e005263, 2018.

[9] C. J. de Carvalho, J. C. B. Marins, C. G. de Lade et al., "Aerobic and resistance exercise in patients with resistant hypertension," Revista Brasileira de Medicina do Esporte, vol. 25, no. 2, pp. 107-111, 2019.

[10] P. C. Hallal and C. G. Victora, "Reliability and validity of the international physical activity questionnaire (IPAQ)," Medicine \& Science in Sports \& Exercise, vol. 36, no. 3, p. 556, 2004.

[11] J. L. Fleg, "Aerobic exercise in the elderly: a key to successful aging," Discovery Medicine, vol. 13, no. 70, pp. 223-228, 2012.

[12] C. Lenfant, A. V. Chobanian, D. W. Jones, and E. J. Roccella, "Seventh report of the joint national committee on the prevention, detection, evaluation, and treatment of high blood pressure (JNC 7)," Hypertension, vol. 41, no. 6, pp. 1178-1179, 2003.

[13] G. Mancia, G. De Backer, A. Dominiczak et al., "2007 guidelines for the management of arterial hypertension," Journal of Hypertension, vol. 25, no. 6, pp. 1105-1187, 2007.

[14] E. I. M. Fransson, L. S. Alfredsson, U. H. de Faire, A. Knutsson, and P. J. M. Westerholm, "Leisure time, occupational and household physical activity, and risk factors for cardiovascular disease in working men and women: the WOLF study," Scandinavian Journal of Public Health, vol. 31, no. 5, pp. 324-333, 2003.

[15] G. Hu, P. Jousilahti, R. Antikainen, and J. Tuomilehto, "Occupational, commuting, and leisure-time physical activity in relation to cardiovascular mortality among finnish subjects with hypertension," American Journal of Hypertension, vol. 20, no. 12, pp. 1242-1250, 2007.

[16] B. Twinamasiko, E. Lukenge, S. Nabawanga et al., "Sedentary lifestyle and hypertension in a periurban area of Mbarara, South western Uganda: a population based cross sectional survey," International Journal of Hypertension, vol. 2018, Article ID 8253948, 8 pages, 2018.

[17] M. Stender, H. W. Hense, A. Döring, and U. Keil, "Physical activity at work and cardiovascular disease risk: results from the MONICA augsburg study," International Journal of Epidemiology, vol. 22, no. 4, pp. 644-650, 1993.

[18] S. Kweon, Y. Kim, M.-j. Jang et al., "Data resource profile: the Korea national health and nutrition examination survey (KNHANES)," International Journal of Epidemiology, vol. 43, no. 1, pp. 69-77, 2014.

[19] C. Medina, I. Janssen, S. Barquera et al., "Occupational and leisure time physical inactivity and the risk of type II diabetes and hypertension among Mexican adults: a prospective cohort study," Scientific Reports, vol. 8, no. 1, p. 5399, 2018.

[20] M. A. Pereira, A. R. Folsom, P. G. McGovern et al., "Physical activity and incident hypertension in black and white adults: the atherosclerosis risk in communities study," Preventive Medicine, vol. 28, no. 3, pp. 304-312, 1999.

[21] A. Holtermann, J. L. Marott, F. Gyntelberg et al., "Occupational and leisure time physical activity: risk of all-cause mortality and myocardial infarction in the copenhagen city heart study. a prospective cohort study," BMJ Open, vol. 2, no. 1, Article ID e000556, 2012.

[22] M. T. Hamilton, D. G. Hamilton, and T. W. Zderic, "Exercise physiology versus inactivity physiology: an essential concept for understanding lipoprotein lipase regulation," Exercise and Sport Sciences Reviews, vol. 32, no. 4, pp. 161-166, 2004.

[23] V. A. Cornelissen and N. A. Smart, "Exercise training for blood pressure: a systematic review and meta-analysis," Journal of the American Heart Association, vol. 2, no. 1, Article ID e004473, 2013.

[24] V. Arija, F. Villalobos, R. Pedret et al., "Physical activity, cardiovascular health, quality of life and blood pressure control in hypertensive subjects: randomized clinical trial," Health and Quality of Life Outcomes, vol. 16, no. 1, p. 184, 2018.

[25] N. L. Chase, X. Sui, D.-C. Lee, and S. N. Blair, "The association of cardiorespiratory fitness and physical activity with incidence of hypertension in men," American Journal of Hypertension, vol. 22, no. 4, pp. 417-424, 2009.

[26] E. G. Ciolac, J. M. Rodrigues da Silva, and M. U. de Rezende, "Physical activity prevents blood pressure increases in individuals under treatment for knee osteoarthritis," Blood Pressure Monitoring, vol. 23, no. 6, pp. 297-300, 2018.

[27] J. U. Gonzales, D. M. Grinnell, M. J. Kalasky, and D. N. Proctor, "Sex-dependent associations between daily physical activity and leg exercise blood pressure responses," Journal of Aging and Physical Activity, vol. 19, no. 4, pp. 306-321, 2011.

[28] A. Boraita Pérez, "Ejercicio, piedra angular de la prevención cardiovascular," Revista Española de Cardiología, vol. 61, no. 5, pp. 514-528, 2008.

[29] R. Fagard, "Exercise is good for your blood pressure: effects of endurance training and resistance training," Clinical and Experimental Pharmacology and Physiology, vol. 33, no. 9, pp. 853-856, 2006.

[30] J. K. Smith, R. Dykes, J. E. Douglas, G. Krishnaswamy, and S. Berk, "Long-term exercise and atherogenic activity of blood mononuclear cells in persons at risk of developing ischemic heart disease," JAMA, vol. 281, no. 18, pp. 1722-1727, 1999.

[31] L. Nelson, M. Esler, G. Jennings, and P. Korner, "Effect of changing levels of physical activity on blood-pressure and haemodynamics in essential hypertension," The Lancet, vol. 328, no. 8505, pp. 473-476, 1986. 
[32] J. P. Fisher and P. J. Fadel, "Therapeutic strategies for targeting excessive central sympathetic activation in human hypertension," Experimental Physiology, vol. 95, no. 5, pp. 572-580, 2010.

[33] M. C. Laterza, L. D. N. J. de Matos, I. C. Trombetta et al., "Exercise training restores baroreflex sensitivity in nevertreated hypertensive patients," Hypertension, vol. 49, no. 6, pp. 1298-1306, 2007.

[34] A. C. Taylor, N. McCartney, M. V. Kamath, and R. L. Wiley, "Isometric training lowers resting blood pressure and modulates autonomic control," Medicine \& Science in Sports \& Exercise, vol. 35, no. 2, pp. 251-256, 2003.

[35] P. J. Millar, S. R. Bray, M. J. MacDonald, and N. McCartney, "The hypotensive effects of isometric handgrip training using an inexpensive spring handgrip training device," Journal of Cardiopulmonary Rehabilitation and Prevention, vol. 28, no. 3, pp. 203-207, 2008.

[36] G. R. Devereux, J. D. Wiles, and I. L. Swaine, "Reductions in resting blood pressure after 4 weeks of isometric exercise training," European Journal of Applied Physiology, vol. 109, no. 4, pp. 601-606, 2010.

[37] P. C. Dempsey, R. N. Larsen, D. W. Dunstan, N. Owen, and B. A. Kingwell, "Sitting less and moving more," Hypertension, vol. 72, no. 5, pp. 1037-1046, 2018.

[38] M. J. Wheeler, D. W. Dunstan, K. A. Ellis et al., "Effect of morning exercise with or without breaks in prolonged sitting on blood pressure in older overweight/obese adults," $\mathrm{Hy}$ pertension, vol. 73, no. 4, pp. 859-867, 2019.

[39] R. L. Wiley, C. L. Dunn, R. H. Cox, N. A. Hueppchen, and M. S. Scott, "Isometric exercise training lowers resting blood pressure," Medicine \& Science in Sports \& Exercise, vol. 24, no. 7, pp. 749-754, 1992.

[40] P. G. Peters, H. M. Alessio, A. E. Hagerman, T. Ashton, S. Nagy, and R. L. Wiley, "Short-term isometric exercise reduces systolic blood pressure in hypertensive adults: possible role of reactive oxygen species," International Journal of Cardiology, vol. 110, no. 2, pp. 199-205, 2006.

[41] J. Casonatto, K. F. Goessler, V. A. Cornelissen, J. R. Cardoso, and M. D. Polito, "The blood pressure-lowering effect of a single bout of resistance exercise: a systematic review and meta-analysis of randomised controlled trials," European Journal of Preventive Cardiology, vol. 23, no. 16, pp. 17001714, 2016. 